\title{
Possible discovery of the r-process characteristics in the abundances of metal-rich barium stars (Research Note)
}

\author{
W. Y. Cui ${ }^{1,2}$, B. Zhang ${ }^{1}$, J. R. Shi ${ }^{3}$, G. Zhao ${ }^{2,3}$, W. J. Wang ${ }^{4}$, and P. Niu ${ }^{1}$ \\ 1 Department of Physics, Hebei Normal University, 20 Nanerhuan East Road, 050024 Shijiazhuang, PR China \\ e-mail: wycui@bao.ac.cn \\ 2 School of Space Science and Physics, Shandong University at Weihai, 264209 Weihai, PR China \\ 3 National Astronomical Observatories, Chinese Academy of Sciences, 20A Datun Road, 100012 Beijing, PR China \\ ${ }^{4}$ Baoding University, Baoding, 071000 Hebei, PR China
}

Received 20 December 2013 / Accepted 22 April 2014

\begin{abstract}
Aims. We study the abundance distributions of a sample of metal-rich barium stars provided by Pereira et al. (2011, A\&A, 533, A51) to investigate the s- and r-process nucleosynthesis in the metal-rich environment.

Methods. We compared the theoretical results predicted by a parametric model with the observed abundances of the metal-rich barium stars.

Results. We found that six barium stars have a significant r-process characteristic, and we divided the barium stars into two groups: r-rich barium stars $\left(C_{\mathrm{r}}>5.0,[\mathrm{La} / \mathrm{Nd}]<0\right)$ and normal barium stars. The behavior of the r-rich barium stars seems more like that of the metal-poor r-rich and CEMP-r/s stars. We suggest that the most possible formation mechanism for these stars is the s-process pollution, although their abundance patterns can be fitted very well when the pre-enrichment hypothesis is included. That we cannot explain them well using the s-process nucleosynthesis alone may be due to our incomplete knowledge on the production of $\mathrm{Nd}$, Eu, and other relevant elements by the s-process in metal-rich and super metal-rich environments (see details in Pereira et al. 2011).
\end{abstract}

Key words. stars: abundances - stars: chemically peculiar - stars: AGB and post-AGB - binaries: general

\section{Introduction}

Heavy elements $(Z>30)$ are produced by neutron-captureprocess nucleosynthesis, which is subdivided into rapid (r-) and slow (s-)processes, depending on the competition between the $\beta$-decay and the next neutron-capture. The main site of the s-process reactions is inside the low-mass stars $(M<$ 3.0 $M_{\odot}$, Gallino et al. 1998; Straniero et al. 2006) during their asymptotic-giant-branch (AGB) phase. The r-process nucleosynthesis is always associated with exploding astrophysical events, such as core-collapse type II supernovae (SNII) or neutron star mergers. The accurate site (sites), however, is (are) still not confirmed for the r-process nucleosynthesis.

According to Burris et al. (2000), 85\% of Ba and 3\% of Eu in the solar system were contributed by the s-process. Thus, Ba and Eu are usually regarded as the representative elements of the s- and r-processes, respectively. More than $80 \%$ of the carbonenhanced metal-poor (CEMP, $[\mathrm{C} / \mathrm{Fe}]>1.0$ and $[\mathrm{Fe} / \mathrm{H}]<-2.0$ ) stars exhibit excesses of the s-process elements (e.g. Ba), that is, they are CEMP-s stars (Aoki et al. 2007). Furthermore, about half of the CEMP-s stars also show significant r-process characteristics (e.g., Eu enrichment), CEMP-r/s stars (Beers \& Christlieb 2005). Different astrophysical sites have been suggested in which the s- and r-process nucleosynthesis occurs, thus the existence of CEMP-r/s stars is confusing. Many mechanisms for their formation have been suggested, but, none of them was able to explain all of their observational characteristics (see details in Jonsell et al. 2006).

Barium stars are a class of chemically peculiar stars that were first described by Bidelman \& Keenan (1951). Barium stars show enhancements of carbon and elements heavier than iron, which are often used to constrain the s-process nucleosynthesis theory (Allen \& Barbuy 2006; Drake \& Pereira 2008). Based on their evolution state, barium stars are divided into two groups: barium giants and barium dwarfs. Barium giants are a group of chemically peculiar $\mathrm{G}$ and $\mathrm{K}$ giants that have evolved into the red giant branch. Fewer barium dwarfs than giants have been confirmed (Gòmez et al. 1997). Barium giants have similar luminosities to red giants (Scalo 1976), which are more luminous than barium dwarfs and less luminous than AGB stars in which the s-process nucleosynthesis is expected to occur (Gallino et al. 1998; Busso et al. 2001). Because of this, the binary hypothesis was required to explain the abundance anomalies of barium stars. In this mechanism, a massive companion that is now a white dwarf invisible in optical observations first produced the s-materials during its AGB evolution phase, and then transferred them to the observed star via Roche-lobe overflow or wind accretion in a binary system.

Pereira et al. (2011) reported detailed chemical abundances and kinematics of a sample of metal-rich barium stars. For convenience, we divided the barium stars into two groups: stars with a metallicity lower than the solar value $([\mathrm{Fe} / \mathrm{H}]<0)$ are 
A\&A 566, A16 (2014)

Table 1. Calculation results of the physical parameters for metal-rich barium stars.

\begin{tabular}{lcccccrr}
\hline \hline Star & {$[\mathrm{Fe} / \mathrm{H}]$} & $\begin{array}{c}\Delta \tau \\
\left(\mathrm{mbarn}^{-1}\right)\end{array}$ & $r$ & $\begin{array}{c}\tau_{0} \\
\left(\mathrm{mbarn}^{-1}\right)\end{array}$ & $C_{\mathrm{s}}$ & $C_{\mathrm{r}}$ & $\chi^{2}$ \\
\hline CD-25 6606 & 0.12 & 0.15 & 0.02 & 0.04 & 0.00971 & 15.3 & 0.69281 \\
HD 46040 & 0.11 & 0.48 & 0.01 & 0.10 & 0.00075 & 10.3 & 9.77175 \\
HD 49841 & 0.21 & 0.13 & 0.02 & 0.03 & 0.03390 & 17.2 & 0.98713 \\
HD 82765 & 0.20 & 0.20 & 0.06 & 0.07 & 0.00154 & 3.4 & 0.13097 \\
HD 84734 & 0.21 & 0.16 & 0.1 & 0.07 & 0.00293 & 13.8 & 0.37039 \\
HD 85205 & 0.23 & 0.32 & 0.01 & 0.07 & 0.00040 & 11.9 & 1.92897 \\
HD 100012 & 0.18 & 0.01 & 0.93 & 0.14 & 0.00044 & 6.5 & 3.50993 \\
HD 101079 & 0.10 & 0.25 & 0.01 & 0.05 & 0.00082 & 7.5 & 0.23397 \\
HD 130386 & 0.16 & 0.17 & 0.22 & 0.11 & 0.00156 & 0.0 & 0.04935 \\
HD 139660 & 0.26 & 0.23 & 0.01 & 0.05 & 0.00167 & 8.4 & 0.22529 \\
HD 198590 & 0.18 & 0.26 & 0.05 & 0.09 & 0.00080 & 3.4 & 0.07676 \\
HD 212209 & 0.30 & 0.12 & 0.01 & 0.03 & 0.03774 & 4.0 & 0.07312 \\
\hline
\end{tabular}

referred to as metal-poor barium stars; the other group of stars with $[\mathrm{Fe} / \mathrm{H}]>0$ is referred to as metal-rich barium stars. Of the 12 stars Pereira et al. (2011), 11 stars were confirmed as barium stars, including seven barium giants and four metal-rich CH subgiants. In addition, Pereira \& Drake (2011) pointed out that no metal-rich barium dwarfs were discovered. In the metalrich sample, abundances of the five neutron-capture elements $\mathrm{Y}$, $\mathrm{Zr}, \mathrm{La}, \mathrm{Ce}$, and Nd were provided (Pereira et al. 2011).

In this paper, we analyze the abundance patterns of the neutron-capture elements of the metal-rich barium stars reported by Pereira et al. (2011) to investigate the s-process nucleosynthesis in the metal-rich environments. The paper is organized as follows: Sect. 2 presents our calculation, results and discussion, and in Sect. 3 our conclusions are drawn.

\section{Results and discussion}

To investigate the s-process nucleosynthesis in metal-rich conditions, we studied the barium star sample provided by Pereira et al. (2011) using the parametric model for low-mass AGB stars (Zhang et al. 2006; Cui et al. 2007, 2010). In our model, the contributions from r-process are also considered. We obtained the theoretical abundance $N_{i}$ of the $i$ th element based on the following formula:

$N_{i}(Z)=C_{\mathrm{s}} N_{i, \mathrm{~s}}+C_{\mathrm{r}} N_{i, \mathrm{r}} 10^{[\mathrm{Fe} / \mathrm{H}]}$,

where $Z$ is the metallicity of the star, $N_{i, \mathrm{~s}}$ and $N_{i, \mathrm{r}}$ are the abundances of the $i$ th element produced by the s- and r-process (per $\mathrm{Si}=10^{6}$ at $\left.Z=Z_{\odot}\right), C_{\mathrm{s}}$ and $C_{\mathrm{r}}$ are the component coefficients representing the contributions of the s- and the r-process. During the calculations, the observed abundances were used to constrain the physical parameters of the neutron-capture nucleosynthesis.

The abundance ratio of barium to europium is particularly sensitive to the relative contributions of the s- and r-process nucleosynthesis for the heavy elements formation. [La/Eu] and $[\mathrm{Ba} / \mathrm{Nd}]$ are also used as important indicators (Mashonkina et al. 2004; Simmerer et al. 2004). Here, we used [La/Nd] as indicator of the relative contributions of the s- and r-process nucleosynthesis for the heavy element productions in a star. In the solar system the heavy elements were formed by the r- and s-process, but different elements have different proportions of the two neutroncapture processes. According to Burris et al. (2000), the main s-process produced $75 \%$ of $\mathrm{La}$ and $47 \%$ of $\mathrm{Nd}$ in the solar system. La is usually regarded as one of the typical s-process elements, while the $\mathrm{Nd}$ production from both the $\mathrm{r}$ - and s-process is similar in the solar system and the exact value depends on the
AGB models (Burris et al. 2000; Arlandini et al. 1999). Thus, different $[\mathrm{La} / \mathrm{Nd}]$ ratios mean different $r$ - and s-process contributions, and the lower the $[\mathrm{La} / \mathrm{Nd}]$ values, the higher the r-process contributions.

Our results are shown in Table 1 . The low $\chi^{2}$ values imply that our theoretical predictions for the yields of neutroncapture elements fit the observational abundances well for the corresponding metal-rich barium stars. This supports the binary hypothesis for the formation of barium stars even in metalrich environments. To confirm this, however, long-term radialvelocity monitoring for these metal-rich barium stars is encouraged. HD 46040 is an exception, which has a high $\chi^{2}$ value, which 9.77175 , this means that the theoretical results cannot fit the observed abundances well. The reason may be its unusually high enrichment of neutron-capture elements especially for La and Ce compared with other stars with similar metallicities. Pereira et al. (2011) suggested that HD 100012 does not belong to the group of barium stars because of its low $[\mathrm{s} / \mathrm{Fe}]$ value, which is similar to the observed values of some normal field stars at this metallicity; here, "s" represents the mean abundance of Y, $\mathrm{Zr}, \mathrm{La}, \mathrm{Ce}$, and Nd. However, the low [s/Fe] value of HD 100012 is mainly due to its low Y abundance; in fact, the other four elements still show enrichment compared with other normal stars (Pereira et al. 2011, see their Fig. 8). Thus, this star is still included in our discussion.

The mean neutron exposure, $\tau_{0}=-\Delta \tau / \ln r$, is a fundamental parameter for the s-process nucleosynthesis, where $\Delta \tau$ is the neutron exposure per thermal pulse in an AGB star, and $r$ is the fraction of material that remains to experience subsequent neutron exposures. Figure 1 shows $\tau_{0}$ and $r$ as a function of $[\mathrm{Fe} / \mathrm{H}]$, where (a) for $\tau_{0}$; and (b) for $r$, respectively. We can see from Fig. 1a that $\tau_{0}$ decreases with increasing $[\mathrm{Fe} / \mathrm{H}]$. Because a higher $\tau_{0}$ value favors the production of heavier elements such as $\mathrm{Ba}, \mathrm{La}$, and $\mathrm{Pb}$ (Busso et al. 2001), this means that the production of s-elements is less efficient in metal-rich environments $([\mathrm{Fe} / \mathrm{H}]>0)$. In other words, the rule of the s-process nucleosynthesis summarized by Gallino et al. (1998) and Busso et al. (2001) is still valid when it extends to metal-rich conditions. From Fig. 1b, we can see that more and more barium stars tend to have lower $r$ values with increasing metallicity. In our metal-rich barium stars, almost all of the $r$ values are lower than 0.1 , except for HD 130386 and HD 100012. This supports the conclusion that single neutron exposure events (usually with $r \leq 0.1$ ) for the s-process nucleosynthesis probable exist throughout the entire metallicity range of the Galaxy evolution (Cui et al. 2013a). Furthermore, the occurrence frequency of single exposure events is very high in metal-rich conditions. 

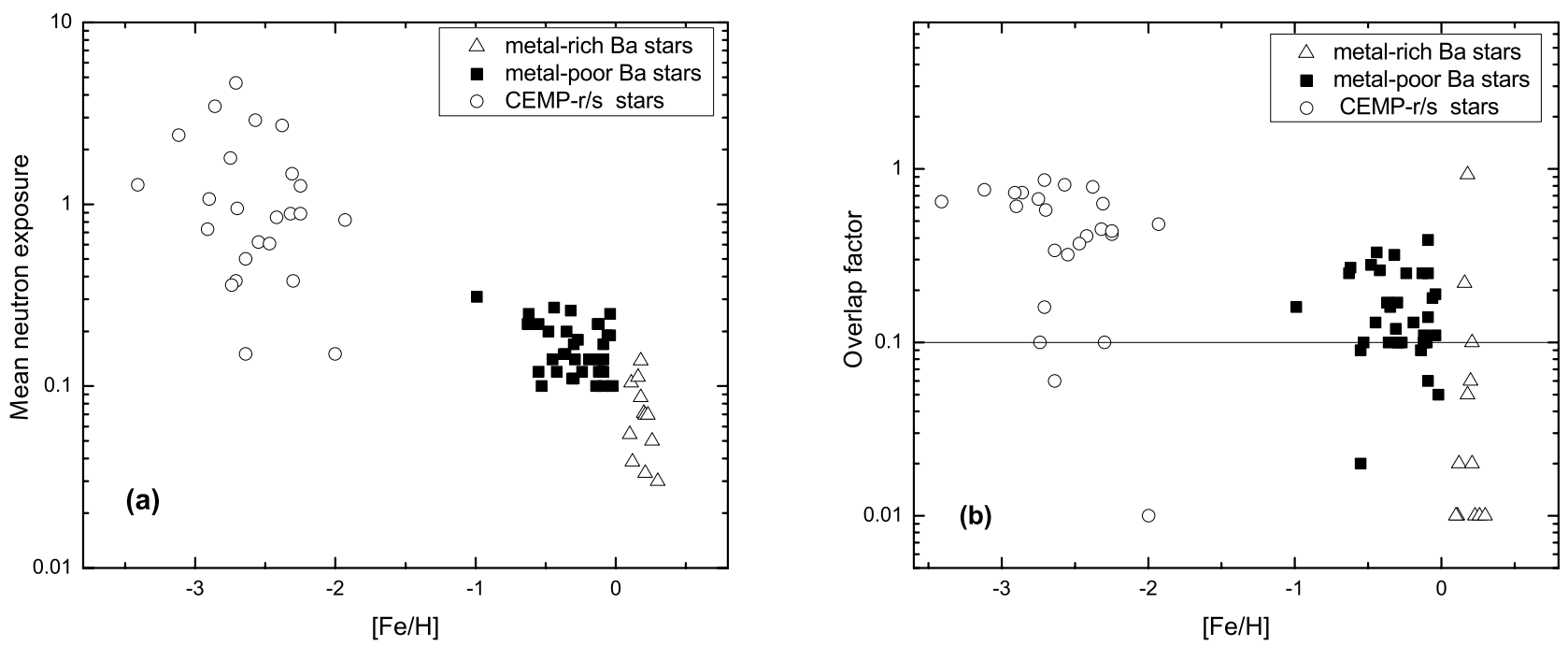

Fig. 1. Physical parameters of s-process nucleosynthesis versus $[\mathrm{Fe} / \mathrm{H}]$ : a) for the mean neutron exposure, $\tau_{0}$; b) for the overlap factors, $r$. We adopted the same CEMP-r/s (open circles) and metal-poor barium (filled squares) star samples as collected by Cui et al. (2013a, and references therein). To use as homogeneous a sample as possible, we show only the sample presented in Allen \& Barbuy (2006) for the metal-poor barium stars. The open triangles represent the metal-rich barium stars adopted from Pereira et al. (2011).

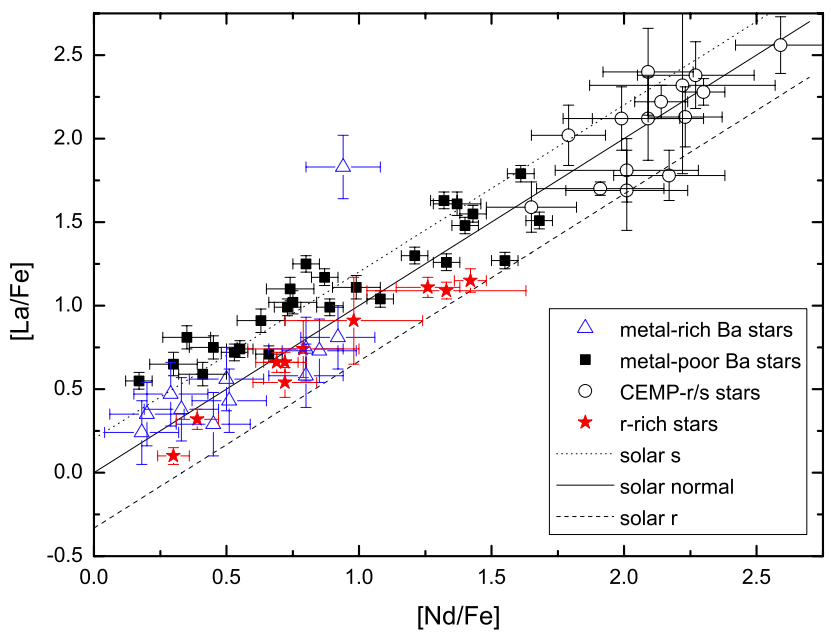

Fig. 2. Abundance ratios $[\mathrm{La} / \mathrm{Fe}]$ versus $[\mathrm{Nd} / \mathrm{Fe}]$. Error bars are also plotted. The symbols are the same as in Fig. 1. Here, r-rich stars (filled stars, including seven r-II stars and three r-I stars) are also presented for comparison. The r-rich stars are taken from the sample collected by Cui et al. (2013b, and references therein). The lines represent the solar $[\mathrm{La} / \mathrm{Nd}]$ values: dotted for solar s, dashed for solar $\mathrm{r}$, and solid for solar normal. (See color version online.)

Table 1 shows that eight barium stars of our sample of 12 metal-rich stars have high $C_{\mathrm{r}}$ values $(>5.0), \mathrm{CD}-25^{\circ} 6606$, HD 46040, HD 49841, HD 84734, HD 85205, HD 100012, HD 101079, and HD 139660. This means that these barium stars have significant r-process abundance characteristics, because the physical parameter, $C_{\mathrm{r}}$, represents the r-process contribution to the neutron-capture elements (see formula 1). This is very confusing because considering their metallicities and observational errors of the element abundances, the $C_{\mathrm{r}}$ values should be lower than about 4.0. In other words, significant r-process abundance characteristics are not expected in metal-rich environments. Figure 2 shows the abundance ratios $[\mathrm{La} / \mathrm{Fe}]$ versus $[\mathrm{Nd} / \mathrm{Fe}]$. In this figure, six metal-rich barium stars with high $C_{\mathrm{r}}$ are in the same region with the r-rich stars, and their $[\mathrm{La} / \mathrm{Fe}]$ and $[\mathrm{Nd} / \mathrm{Fe}]$ ratios also show a highly positive correlation. It

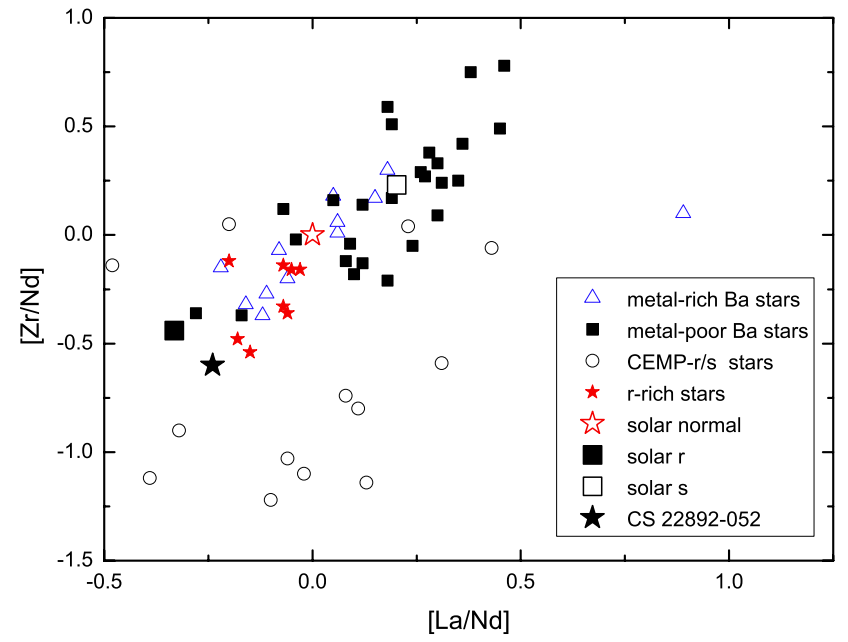

Fig. 3. Abundance ratios $[\mathrm{Zr} / \mathrm{Nd}]$ versus $[\mathrm{La} / \mathrm{Nd}]$. The symbols are the same as in Figs. 1 and 2. The large symbols represent solar values: filled square for solar $r$, open squares for solar s, and open stars for solar normal, except for the filled star, which represents the typical r-II star CS 22892-052. (See color version online.)

seems that these six metal-rich barium stars belong to an independent group because they have similar abundance characteristics with the r-rich stars. For the metal-poor barium stars, however, this is not the case (Cui et al. 2013a, see their Tables 1, 2).

The abundance ratios $[\mathrm{Zr} / \mathrm{Nd}]$ vs. $[\mathrm{La} / \mathrm{Nd}]$ are presented in Fig. 3 and show that these six metal-rich barium stars with high $C_{\mathrm{r}}$ and the r-rich stars are distributed in the same area, $-0.4<[\mathrm{La} / \mathrm{Nd}]<0$ and $-0.7<[\mathrm{Zr} / \mathrm{Nd}]<0$. This further supports that these barium stars belong to an independent group. It is interesting that the $[\mathrm{Zr} / \mathrm{Nd}]$ ratio of the solar r-process is higher than in some r-rich stars, such as CS 22892-052, which is a typical $\mathrm{r}-\mathrm{II}$ star $([\mathrm{Eu} / \mathrm{Fe}]>1)$, where no contribution from the weak r-process is expected (Travaglio et al. 2004; Ishimaru et al. 2005; Arcones \& Montes 2011). The reason is that solar $\mathrm{Zr}$ is enriched by massive stars through the weak r- and s-process nucleosynthesis, but the weak s-process does not work in extremely metal-poor environments (The et al. 2000). Studying these stars 


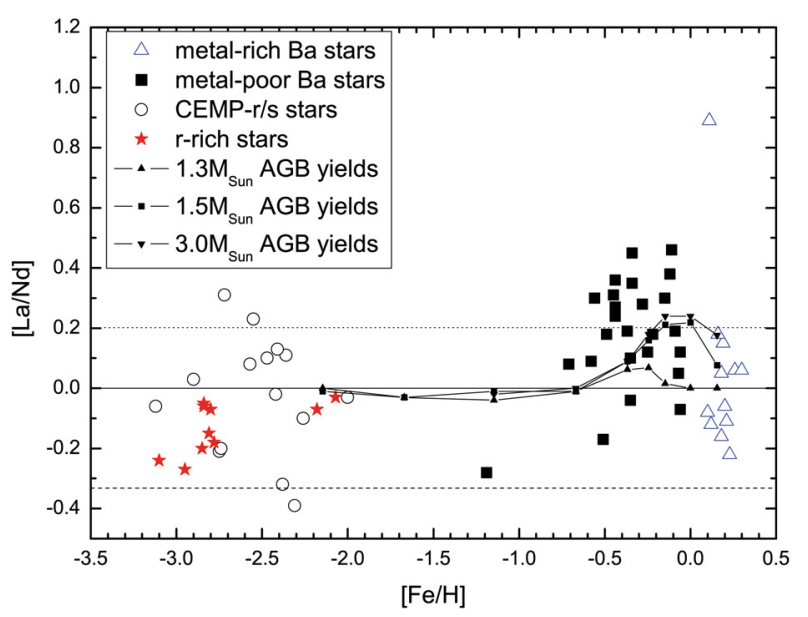

Fig. 4. Abundance ratios $[\mathrm{La} / \mathrm{Nd}]$ versus $[\mathrm{Fe} / \mathrm{H}]$. The symbols are the same as in Fig. 1. The small symbols represent the AGB model yields of Cristallo et al. (2011). (See color version online.)

might shed some light on the neutron-capture process including both r- and s-process nucleosynthesis, especially in metal-rich conditions.

Figure 4 shows the abundance ratios $[\mathrm{La} / \mathrm{Nd}]$ as a function of $[\mathrm{Fe} / \mathrm{H}]$ for metal-rich and metal-poor barium stars and CEMP-r/s stars. Combing Fig. 4 with Table 1, we found that the metal-rich barium stars with high $C_{\mathrm{r}}$ values have a similar characteristic, $[\mathrm{La} / \mathrm{Nd}]<0$, except for HD 139660 . Because the $[\mathrm{La} / \mathrm{Nd}]$ ratios can be used as indicators of the relative contributions of s- and $r$-process nucleosynthesis for the heavy element productions in a star, we call these barium stars with $[\mathrm{La} / \mathrm{Nd}]<0$ and $C_{\mathrm{r}}>5.0$ r-rich barium stars and the barium stars with $[\mathrm{La} / \mathrm{Nd}]>0$ normal barium stars. Figure 4 shows that the r-rich barium stars are spread in a similar $[\mathrm{La} / \mathrm{Nd}]$ range to those of the metalpoor r-rich stars and part of the CEMP-r/s stars. Furthermore, even if the effects of different initial mass were considered, the AGB model yields of Cristallo et al. (2011) are also unable to produce negative $[\mathrm{La} / \mathrm{Nd}]$ values such as $[\mathrm{Fe} / \mathrm{H}]>-0.6$. Thus, the r-rich metal-rich barium stars seem to have same abundance behavior as r-rich or CEMP-r/s stars. Of course, more observations to measure the abundances of $\mathrm{Ba}$ and $\mathrm{Eu}$ are needed.

Figure 4 also shows that the CEMP-r/s stars are spread in a wide range of $[\mathrm{La} / \mathrm{Nd}]$ about from -0.4 to 0.3 . Much effort has been made to solve the problem of the origin of CEMP-r/s stars (Cui et al. 2010, 2013a; Bisterzo et al. 2009). Commonly, a mixed origin is assumed that the observed CEMP-r/s star had been successively polluted by the s- and r-process materials, although the accurate site for the r-process nucleosynthesis is still uncertain. In addition, the AGB models also predict negative $[\mathrm{La} / \mathrm{Nd}]$ values when different efficiencies of the ${ }^{13} \mathrm{C}$ neutron source are included in the calculations, especially in extremely metal-poor conditions $([\mathrm{Fe} / \mathrm{H}] \leq-2.0$ ) (Busso et al. 2001; Straniero et al. 2006; Zhang et al. 2006; Bisterzo et al. 2010), but this does not work in metal-rich conditions (Bisterzo et al. 2010, see their Fig. 5, bottom panel). When a mixed contribution of the r- and s-process nucleosynthesis was considered, the observed abundance patterns of CEMP-r/s stars were able to be fitted well by the theoretical predictions (Cui et al. 2007, 2010, 2013a; Bisterzo et al. 2009). This is also correct for almost all metal-poor and metal-rich barium stars including all r-rich metal-rich barium stars. There are four metal-poor barium stars, HD 749, HD 5424, HD 123396, and HD 223938, which have negative $[\mathrm{La} / \mathrm{Nd}]$ values but low $C_{\mathrm{r}}$, they are only a small part of our 26 metal-poor barium star sample. None of them was able to be fitted well using the mixed mechanism. Two of them, HD 5424, HD 123396, have lower values of [La/Nd], -0.17 and -0.28 , and also have higher $\chi^{2}, 10.52647$ and 13.98755, respectively. The reason is unclear, however.

R-rich stars are commonly suggested to be formed from molecular clouds that had been polluted by SNII. For the CEMP$\mathrm{r} / \mathrm{s}$ stars, the favored mechanism is pre-enrichment: the first formed r-rich star is polluted by the s-process material through mass-transfer from its former AGB companion in a binary system. But this was challenged by Lugaro et al. (2012) because of the following reasons: first, it is difficult to explain the linear correlation observed between $[\mathrm{Ba} / \mathrm{Fe}]$ and $[\mathrm{Eu} / \mathrm{Fe}]$ in CEMP-r/s stars; the initial $[\mathrm{r} / \mathrm{Fe}]$ does not affect the final $[\mathrm{Ba} / \mathrm{Fe}]$ (Bisterzo et al. 2009). Second, it is difficult to understand that there are fewer observed $\mathrm{r}$-II stars $([\mathrm{Eu} / \mathrm{Fe}]>1)$ than CEMP-r/s stars, if CEMP-r/s stars were suggested to evolve from r-II stars in binary systems. Finally, the metallicity distribution for the two groups is different (see details in Lugaro et al. 2012). For these reasons, Lugaro et al. (2012) proposed a new r/s-process as a possible choice for CEMP-r/s star formation. This process has features that mix or superpose the s- and r-process. This might be a choice for the origin of the r-rich barium stars. However, the inhomogeneity of the interstellar medium is not expected in such a environment with $[\mathrm{Fe} / \mathrm{H}]>0$. Allen et al. (2012) proposed CEMP-r/s stars have the same origin as CEMP-s stars. In other words, the additional r-process site is not needed for the formation of CEMP-r/s stars. The reason that the s-process cannot explain the abundance patterns of the CEMP-r/s stars alone is our incomplete knowledge of the Eu production by the s-process nucleosynthesis at low metallicity. It may be the most possible formation mechanism for the r-rich barium stars. It is also very important to include Eu and other relevant elements in studying the metal-rich barium stars.

In this work, $\mathrm{Nd}$ and $\mathrm{La}$ abundances are very important to determine whether a star belongs to the class of r-rich barium stars. As a typical star of the sample, Pereira et al. (2011) presented abundance uncertainties for the star CD-25 6606 (see their Table 7). Using the method presented by Aoki et al. (2001) and Zhang et al. (2006), we discuss the uncertainty of the model parameter $C_{\mathrm{r}}$, which is propagated from the abundance uncertainties of $\mathrm{Nd}$ and La. Figure 5 shows the calculated abundance ratios $[\mathrm{Nd} / \mathrm{Fe}]$ and $[\mathrm{La} / \mathrm{Fe}]$ as a function of the r-process component coefficient $C_{\mathrm{r}}$ in a model with $\Delta \tau=0.15, r=0.02$ and $C_{\mathrm{s}}=0.00971$. These calculated results are compared with the observed abundances ratios of CD- $25^{\circ} 6606$. There is only one region of overlap in Fig. 5, $C_{\mathrm{r}}=15.3_{-2.0}^{+6.3}$, in which the observed ratios of both $[\mathrm{Nd} / \mathrm{Fe}]$ and $[\mathrm{La} / \mathrm{Fe}]$ can be accounted for well. The bottom panel displays the $\chi^{2}$ values calculated by the parametric model, and the minimum is $\chi^{2}=0.69281$ at $C_{\mathrm{r}}=15.3$. It can be seen that the physical parameter $C_{\mathrm{r}}$ can be constrained in a reasonable region. We used $C_{\mathrm{r}} \geq 5.0$ as the reference value to judge whether a star belonged to the r-rich barium star group. In fact, this value is lower than 2.0 in most normal barium stars (Cui et al. 2013a).

\section{Conclusions}

Using a parametric model, we studied the sample of metalrich barium stars provided by Pereira et al. (2011). Our results show that more than half of the sample stars need high $C_{\mathrm{r}}$ values to be best fitted. In other words, these barium stars 
W. Y. Cui et al.: Metal-rich barium stars $(R N)$

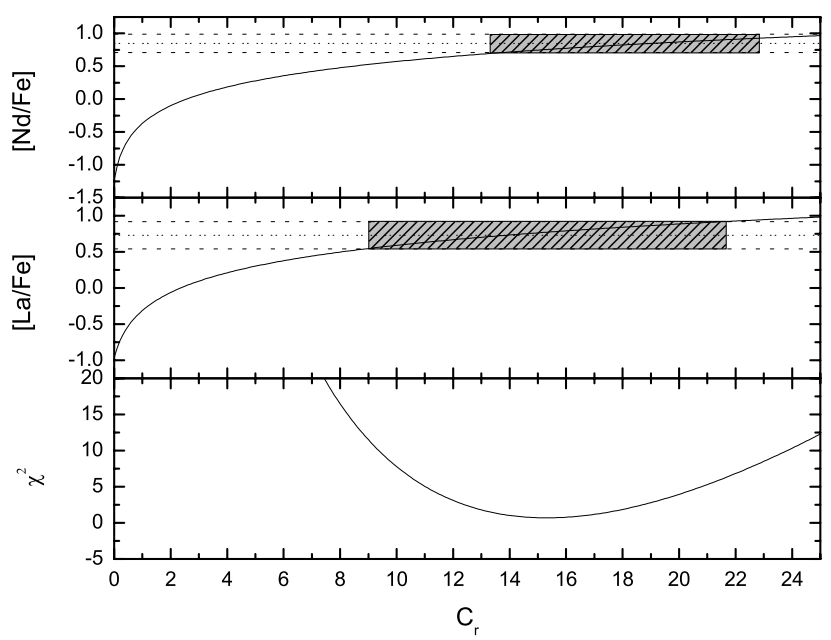

Fig. 5. Abundance ratios $[\mathrm{Nd} / \mathrm{Fe}]$ (top), $[\mathrm{La} / \mathrm{Fe}]$ (middle), and $\chi^{2}$ (bottom), as a function of the r-process component coefficient in a model with $\Delta \tau=0.15, r=0.02, C_{\mathrm{s}}=0.00971$. Solid curves refer to the theoretical results, horizontal dotted lines to the observational results with errors represented by dashed lines. The shaded area illustrates the region allowed for the theoretical model.

have a significant r-process characteristic. Based on this, we divided the barium stars into two groups: the r-rich barium stars $\left(C_{\mathrm{r}}>5.0,[\mathrm{La} / \mathrm{Nd}]<0\right)$ and normal barium stars. The r-rich barium stars seem to have the same abundance behavior as the metal-poor r-rich and CEMP-r/s stars. Although their abundance patterns were fitted very well using a mixed mechanism such as pre-enrichment, we still think that the most plausible formation mechanism for these stars is the s-process pollution. That we cannot explain them well using the s-process nucleosynthesis alone is maybe due to our incomplete knowledge on the production of $\mathrm{Nd}$ and other relevant elements by the s-process in metalrich and super metal-rich environments (see details in Allen et al. 2012).

Obviously, large uncertainties still remain in this topic, and a full understanding of these r-rich barium stars will depend on the abundance information of $\mathrm{Ba}$ and Eu. More in-depth theoretical studies of the s-process nucleosynthesis will also help to shed light on the origin of r-rich barium stars and CEMP-r/s stars.
Acknowledgements. We acknowledge the referee for helpful comments and suggestions that significantly improved the paper. This work was supported by the National Natural Science Foundation of China under grants U1231119, 11273011, 11021504, 11390371, 11003002, 10973016, the China Post-doctoral Science Foundation under grant 2013M531587, the Natural Science Foundation of Hebei Province under grants A2011205102, A2011205067 and the Program for Excellent Innovative Talents in University of Hebei Province under grant CPRC034, the Natural Science Foundation of Hebei Provincial Education Department under grant Z2010168.

\section{References}

Allen, D. M., \& Barbuy, B. 2006, A\&A, 454, 917

Allen, D. M., Ryan, S. G., Rossi, S., Beers, T. C., \& Tsangarides, S. A. 2012, A\&A, 548, A34

Arcones, A., \& Montes, F. 2011, ApJ, 731, 5

Arlandini, C., Käppeler, F., Wisshak, K., et al. 1999, ApJ, 525, 886

Aoki, W., Ryan, S. G., Norris, J., Beers, T. C., \& Ando, H., et al. 2001, ApJ, 561, 346

Aoki, W., Beers, T. C., Christlieb, N., et al. 2007, ApJ, 655, 492

Beers, T. C., \& Christlieb, N. 2005, ARA\&A, 43, 531

Bidelman, W. P., \& Keenan, P. C. 1951, ApJ, 114, 473

Bisterzo, S., Gallino, R., Straniero, O, \& Aoki, W. 2009, PASA, 26, 314

Bisterzo, S., Gallino, R., Straniero, O., Cristallo, S., \& Käppeler, F. 2010, MNRAS, 404, 1529

Burris, D. L., Pilachowski, C. A., Armandroff, T., et al. 2000, ApJ, 544, 302

Busso, M., Gallino, R., Lambert, D. L., Travaglio, C., \& Smith, V. V. 2001, ApJ, 557, 802

Cristallo, S., Piersanti, L., Straniero, O., et al. 2011, ApJS, 197, 17

Cui, W.-Y., Zhang, B., Ma, K., \& Zhang, L. 2007, ApJ, 657, 1037

Cui, W.-Y., Zhang, J., Zhu, Z.-Z., \& Zhang, B. 2010, ApJ, 708, 51

Cui, W.-Y., Shi, J, Geng, Y., et al. 2013a, Ap\&SS., 346, 477

Cui, W.-Y., Sivarani, T., \& Christlieb, N. 2013b, A\&A, 558, A36

Drake, N. A., \& Pereira, C. B. 2008, AJ, 135, 1070

Gallino, R., Arlandini, C., Busso, M., et al. 1998, ApJ, 497, 388

Gomez, A. E., Luri, X., Grenier, S., et al. 1997, A\&A, 319, A881

Ishimaru, Y., Wanajo, S., Aoki, W., Ryan, S. G., \& Prantzos, N. 2005, Nucl. Phys. A, 758, 603

Jonsell, K., Barklem, P. S., Gustafsson, B., et al. 2006, A\&A, 451, 651

Lugaro, M., Karakas, A. I., Stancliffe, R. J., \& Rijs, C. 2012, ApJ, 747, 2

Mashonkina, L. I., Kamaeva, L. A., Samotoev, V. A., \& Sakhibullin, N. A. 2004, Astron. Rep., 48, 185

Pereira, C. B., \& Drake, N. A. 2011, AJ, 141, 79

Pereira, C. B., Sales Silva, J. V., Chavero, C., Roig, F., \& Jilinski, E. 2011, A\&A, 533, A51

Scalo, L. 1976, ApJ, 206, 474

Simmerer, J., Sneden, C., Cowan, J. J., et al. 2004, 617, 1091

Straniero, O., Gallino, R., \& Cristallo, S. 2006, Nucl. Phys. A, 777, 311

The, L. S., El Eid, M. F., \& Meyer, B. S. 2000, ApJ, 533, 998

Travaglio, C., Gallino, R., Arnone, E., et al. 2004, ApJ, 601, 864

Zhang, B., Ma, K., \& Zhou, G. 2006, ApJ, 642, 1075 